Section 12. Electrical and electronical properties

\title{
Semiconductor clusters in the sol-gel process: synthesis and properties of CdS nanocomposites
}

\author{
Lubomir Spanhel, Ertugrul Arpac and Helmut Schmidt \\ Institut für Neue Materialien, W-6600 Saarbrücken, Germany
}

\begin{abstract}
CdS nanocomposites (CdS cluster sizes $<5 \mathrm{~nm}$ ) were prepared via multifunctional inorganic-organic sol-gel processing. In CdS sols as precursors, the CdS clusters are carrying inorganic components as stabilizing centers, along with bifunctional ligands acting as inorganic and organic network formers. Hydrolysis and condensation produces an inorganic skeleton yielding viscous liquids useful to prepare films or monoliths. The final organic cross-linking at $T<100^{\circ} \mathrm{C}$ results in optically transparent materials. The nature of stabilizing centers and the starting synthesis conditions influence strongly the resulting electronic properties. $\mathrm{Cd}^{2+}$ complexed by mercapto compounds gives smaller cluster sizes with a narrower distribution than $\mathrm{Cd}^{2+}$ complexed with amine or carboxylate groups. Mercaptosilane-stabilized CdS clusters synthesized via cleavage of bis triethoxy silyl propyl tetrasulfides show an enhanced room temperature photoluminescence compared with similarly stabilized clusters prepared through $\mathrm{H}_{2} \mathrm{~S}$ exposure.
\end{abstract}

\section{Introduction}

Semiconductors of reduced dimensionality are known to possess size dependent electronic properties based on the quantum mechanical 'particle in a box'-like exciton confinement effects [1-3]. During studies on doped glasses [4] and colloidal semiconductors $[5,6]$ it became evident that small clusters might serve as a potentially interesting candidates for tunable linear and non-linear optics (NLO). The role of chemistry to control NLO efficiency determining factors such as the volume normalized oscillator strength (via cluster size), wavelength selectivity (size distribution), excited state relaxation dynamics and photostability (surface modifications), and local field enhancement (refractive index gradients) is decisive [710]. To take into account these factors in the development of workable devices by soft chemistry routes, variable and flexible processing conditions are needed and the sol-gel technique therefore offers appropriate means. Thus, semiconductor quantum dots have been prepared by various sol-gel routes. In ref. [11], the consolida- tion of $\mathrm{CdS}$ or $\mathrm{ZnO}$ clusters to optically transparent materials has been demonstrated and in refs. $[12,13]$ an 'in situ' CdS cluster growth through $\mathrm{H}_{2} \mathrm{~S}$ exposure of $\mathrm{Cd}^{2+}$-doped silica and alumina sol-gel glasses was performed. These methods do not allow particle growth and stabilization process independent of the incorporation of the particle into a matrix or consolidation to a solid.

As shown in textbooks of colloid chemistry, a variety of means for the stabilization of colloids is known. If these stabilizers can be used for consolidation, a new concept of preparing colloidal composites becomes possible. The route of preparing colloidal composites by use of bifunctional stabilizers was investigated. This route seems to be feasible to design materials with a high abrasion and mechanical resistance [14,15], to fabricate coatings with a very low surface energy [16], to functionalize polysiloxanes and silicones $[17,18]$, as well as to use it for synthesizing semiconductor quantum dots. The goal of this study was to prepare semiconductor sol precursors in which the clusters are carrying multifunctional polymerizable ligands, and subsequentally 
to transform them to nanocomposites by ligand reactions. The detailed conception of this route is given in fig. 1.

The closest layer around the QD represents functional inorganic groups (X) acting as the cluster stabilizer and the surface modifier (starting sol). These groups are covalently linked to the inorganic units via short organic chains (three $\mathrm{CH}_{2}$ groups at most). In order to end up with groupings able to form inorganic networks and/or (in addition to this) organic chains, it is conveniant to use bifunctional silanes of the general formula

$\mathrm{L}-\left(\mathrm{CH}_{2}\right)_{3} \stackrel{\mathrm{Si}}{\mathrm{S}-}$

where $\mathrm{L}$ represents functional groupings either being ask to form complexes or bonds to inorganics (e.g., $\mathrm{L}=-\mathrm{NH}_{2},-\mathrm{SH},-\mathrm{PO}_{3},-\mathrm{SO}_{3}$ ) or to polymerize (e.g., $\mathbb{L}=$-vinyl, -methacroyl, -epoxy). The $-\mathrm{Si}(\mathrm{OR})_{3}$ grouping is able to undergo conventional sol-gel reactions.

Quantum Dot - Nanocomposites

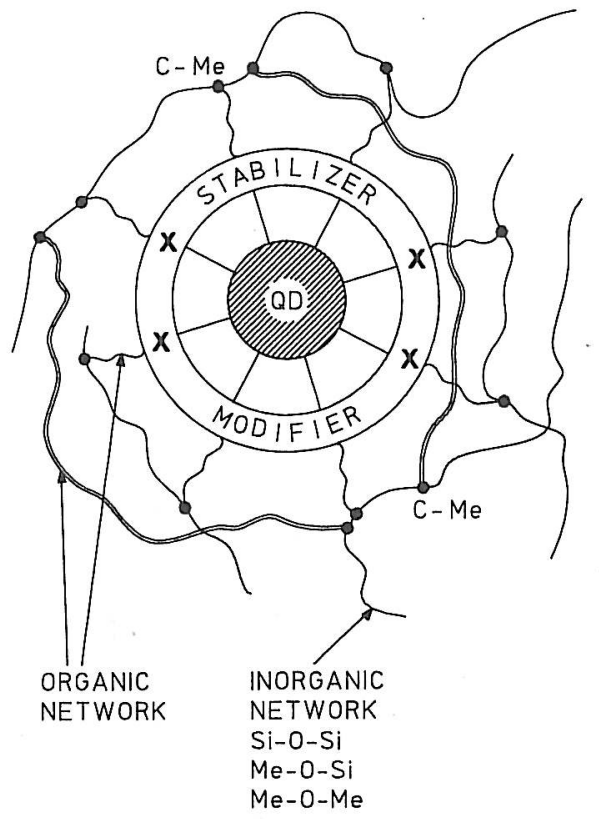

Fig. 1. Scheme of the quantum dot composite preparation concept by use of bifunctional stabilizers. $\mathrm{X}$, ligand acting as link to $\mathrm{CdS}$; $\mathrm{C}-\mathrm{Me}$, hybrid bifunctional group, e.g., $\mathrm{NH}_{2}-$ $\mathrm{Si}(\mathrm{OR})_{3}\left(\right.$ with $\left.-\mathrm{NH}_{2}=\mathrm{X}\right)$.

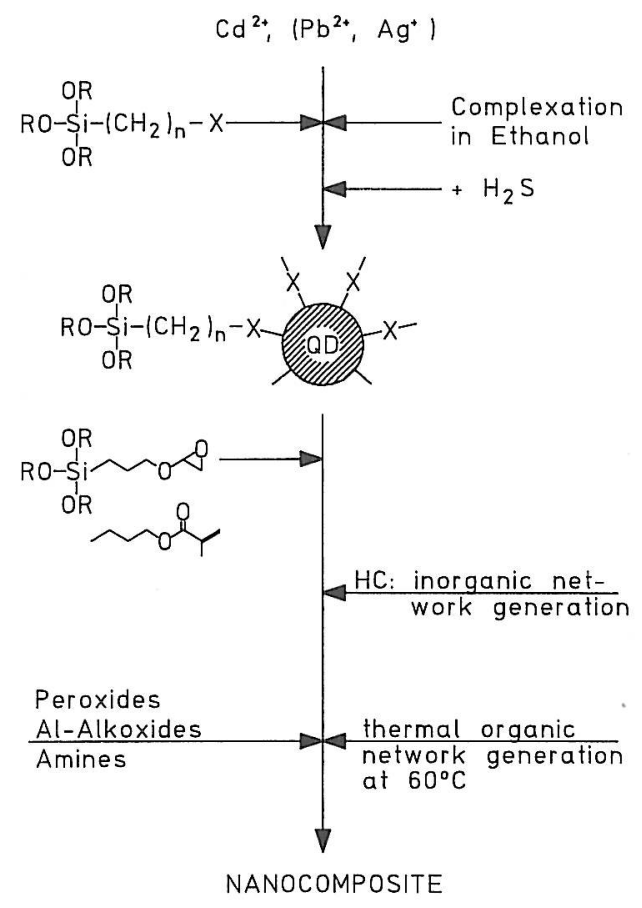

Fig. 2. General reaction route to $\mathrm{CdS}$ quantum dot nano composites; $\mathrm{HC}$, hydrolysis and condensation.

This paper focusses on the preparation route to CdS nanocomposites and on the characterization of optical properties by UV-VIS- and photoluminescence spectroscopy.

\section{Experimental}

The processing of CdS nanocomposites is summarized in fig. 2. Cadmium was introduced as an ethoxide (prepared according to the procedure for making up water free zinc ethoxide [11]), mixed in ethanol with a functionalized triethoxysilane (the organotriethoxysilanes used in the preparations are shown in fig. 3) and exposed to $\mathrm{H}_{2} \mathrm{~S}$ gas under nitrogen atmosphere. The CdS synthesis from bis triethoxysilyl propyl tetrasulfide occurred under ambient laboratory conditions using freshly prepared $0.1 \mathrm{M}$ sodiumboranate solution (the tetrasulfide to boranate ratio was 1 ). The molar silane/Cd ratio was 20 and the resulting CdS sols contained a $50 \%$ excess of $\mathrm{Cd}$. 

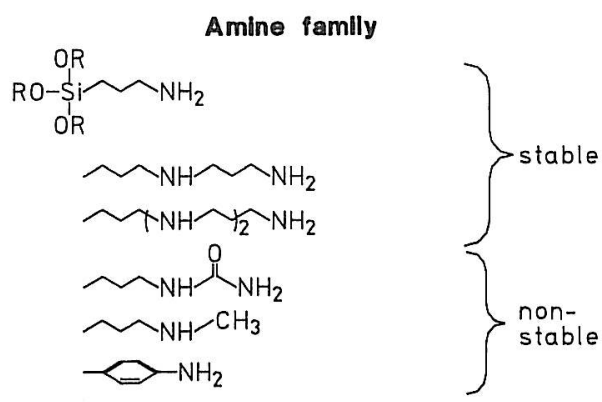

Thiole famlly
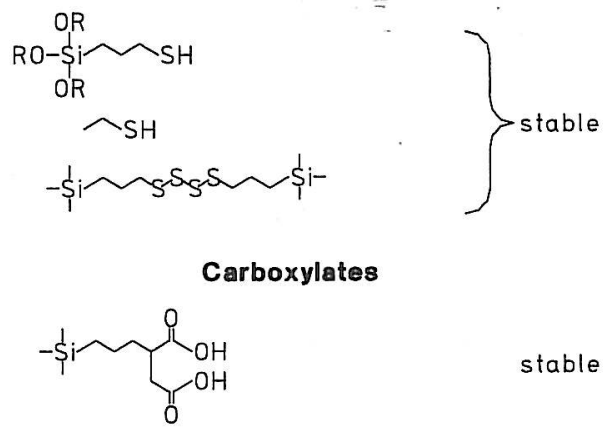

stable

Fig. 3. Variety and effect of bifunctional ligands used in CdS sol stabilization.

In the second step, glycidyloxypropyl trimethoxy silane or methacroylpropyl trimethoxy silane were added (the ratio silane to $\mathrm{Cd}$ was 100).
Subsequentally, hydrolysis and condensation under $\mathrm{CO}_{2}$ atmosphere was performed, the $\mathrm{Si}(\mathrm{OR})_{3} / \mathrm{H}_{2} \mathrm{O}$ ratio being 2 . After the solvent removal, organic polymerization at $60^{\circ} \mathrm{C}$ was performed. Aluminium alkoxides, aminopropyl-triethoxy silane or azobisisobytyronitrile were used as initiators. The viscous liquids were cured in moulds for monolith preparation.

Optical absorption spectra were taken with an Omega 30 spectrophotometer (Bruins Instruments) and the fluorescence spectra were recorded with a 3010 Hitachi spectrofluorometer. Cluster sizing was carried out using a JEOL 200CX transmission electron microscope and photon correlationspectroscopy (ALV-5000).

\section{Results}

Figure 3 shows differently functionalized organoethoxy silanes chosen to stabilize CdS clusters. Within the amine family, monoamine-, diamineand triamine groups gave stable sols. Urea-, methylamine- and aniline functions yield turbid precipitates. One might speculate that the decreased stability in the latter cases is caused by the electron pull or steric effects leading to weaker cadmium complex stabilities, and as a
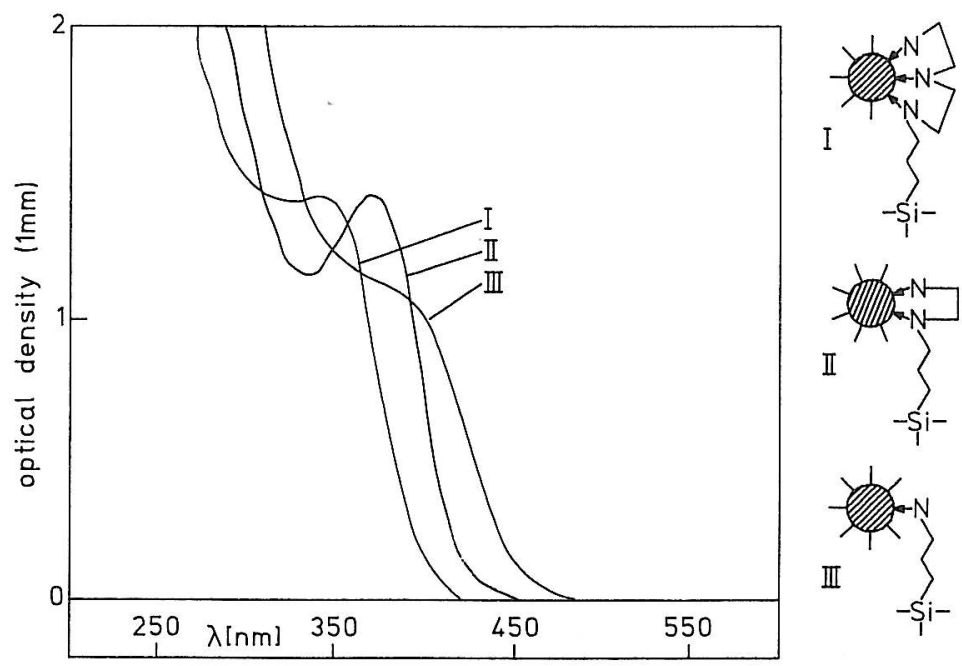

Fig. 4. The effect of amine-derived stabilizers on the optical spectra of CdS cluster sols. 
consequence, to the formation of larger aggregates by agglomeration or precipitation.

The thiole family and the succinic carboxygroups both resulted in stable sols. In the stable case, all sols were completely clear. A special case is represented by the use of tetrasulfide containing silanes which have been employed as a $\mathrm{H}_{2} \mathrm{~S}$ generator as well as for stabilizing.

Figure 4 depicts optical absorption spectra of three different amino stabilized stable CdS sols, characteristic of clusters $\leqslant 3 \mathrm{~nm}$ in size, before connecting them by polycondensation or polymerization. One recognizes that monoamine modified sols absorb at longer wavelengths, indicating a smaller band gap characteristic of cluster sizes of about $4 \mathrm{~nm}$, larger than the approximately 2 $\mathrm{nm}$ clusters in the triamine-stabilized sols. The amine/Cd ratio is the same in the both cases. Since it can be assumed that complex formation power of the triamine group is stronger than that of monoamine, one can conclude that the CdS cluster sizes produced are smaller if $\mathrm{Cd}$ amino complexes are stronger. The pronounced excitonic band at $370 \mathrm{~nm}$ in the diamine case indicates narrower size distribution than in both other cases. All three samples exhibit intense room temperature photoluminescence with a quantum yield of about $10 \%$. While the photoluminescence colour of triamine sols is blue, monoamine sols are green-yellow, luminescing when exposed to UV which also indicates larger particle sizes in monoamine sols.

The effect of mercapto ligands on the cluster optical absorption and photoluminescence spectra is illustrated in fig. 5. Mercaptopropyl silanestabilized clusters possess remarkable strong excitonic band at $302 \mathrm{~nm}$ and a weak luminescence band with a maximum at $450 \mathrm{~nm}$ (cluster sizes $<2 \mathrm{~nm}$ ). In the presence of mercaptomethyl silane, broader size distributions exist, as can be recognized from the longer absorption tail with an onset at $430 \mathrm{~nm}$. The spectra (3) result from samples prepared via boranate attack on triethoxysilylpropyl tetrasulfide to release $\mathrm{H}_{2} \mathrm{~S}$ (reductive cleavage of $\mathrm{S}-\mathrm{S}$ bonds) and to induce $\mathrm{CdS}$ cluster growth. Although the clusters produced are probably stabilized similar to the mercaptopropyl clusters (1), their spectra are redshifted and unstructured; that means larger and broader distributed cluster sizes are produced via the boranate procedure. However, the boranate method produces clusters luminescing five times more intensely. The quantum yield is the same order magnitude as observed with amine derived samples.

Comparing figs. 4 and 5, one can conclude that mercapto-based cluster synthesis offers smaller and narrower distributed CdS quantum dots than

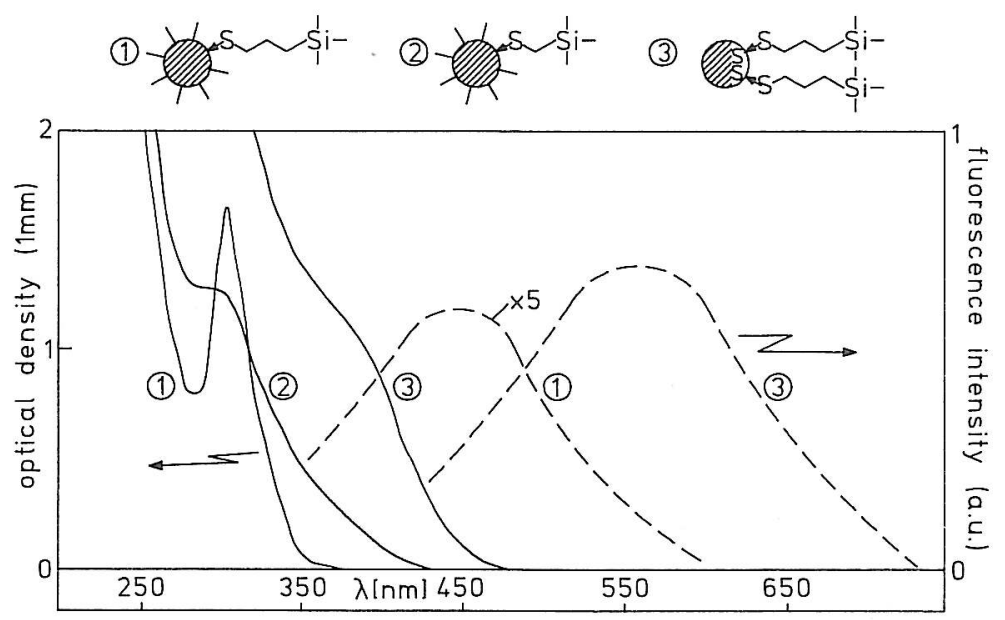

Fig. 5. The effect of thiole-derived stabilizers on the CdS cluster optical absorption and photoluminescence spectra. 
the amine-based preparations. The carboxylatestabilized CdS exhibits spectroscopic properties similar to those of via boranate preparation.

It is known from the literature that broader size distributions broaden or completely screen excitonic peaks within the strong confinement regime. From the measured spectra, only a rough spectroscopic size estimate can be made.

Note, there are no quantum-mechanical models available yet which would allow correlation of TEM with spectroscopic data covering the entire quantum size regime of semiconductor quantum dots. Monosized quantum dots are required for fitting the spectroscopic data but even a high resolution TEM does not serve as a trustful method to determine cluster sizes in the range of some $\mathrm{nm}$ due to experimental difficulties and possible artifacts; however, TEM data of the clusters roughly confirm the spectroscopic estimates.

The generration of organic polymeric network by cross-linking the ligands at $60^{\circ} \mathrm{C}$ does not produce significant changes in electronic properties. The resulting optically transparent monoliths (or sol-gel coatings) generally show very similar spectroscopic properties to the colloidal sols. In some cases, differences are noted depending on initiator used for polymerization.

As already discussed in the experimental section, monoliths could be prepared from all compositions. Figure 6 shows differently shaped monoliths with different CdS cluster sizes. With increasing cluster size, the monoliths are more yellowish colored. The propylmercapto-stabilized CdS precursors (excitonic peak at $300 \mathrm{~nm}$ ) gave colourless samples, whereas the carboxylate stabilized ones resulted in the most intensively yellow colours (bulk CdS crystals without confinement are orange).

\section{Conclusions}

Inorganic-organic sol-gel processing of semiconductor nanocomposites has been presented. It has been shown that functionalized organoalkoxy silanes can be used as a stabilizers and surface modifiers for semiconductor quantum dots. The nature of the chemical environment of different quantum dots can be widely varied, and ligands other than those used in the present paper may

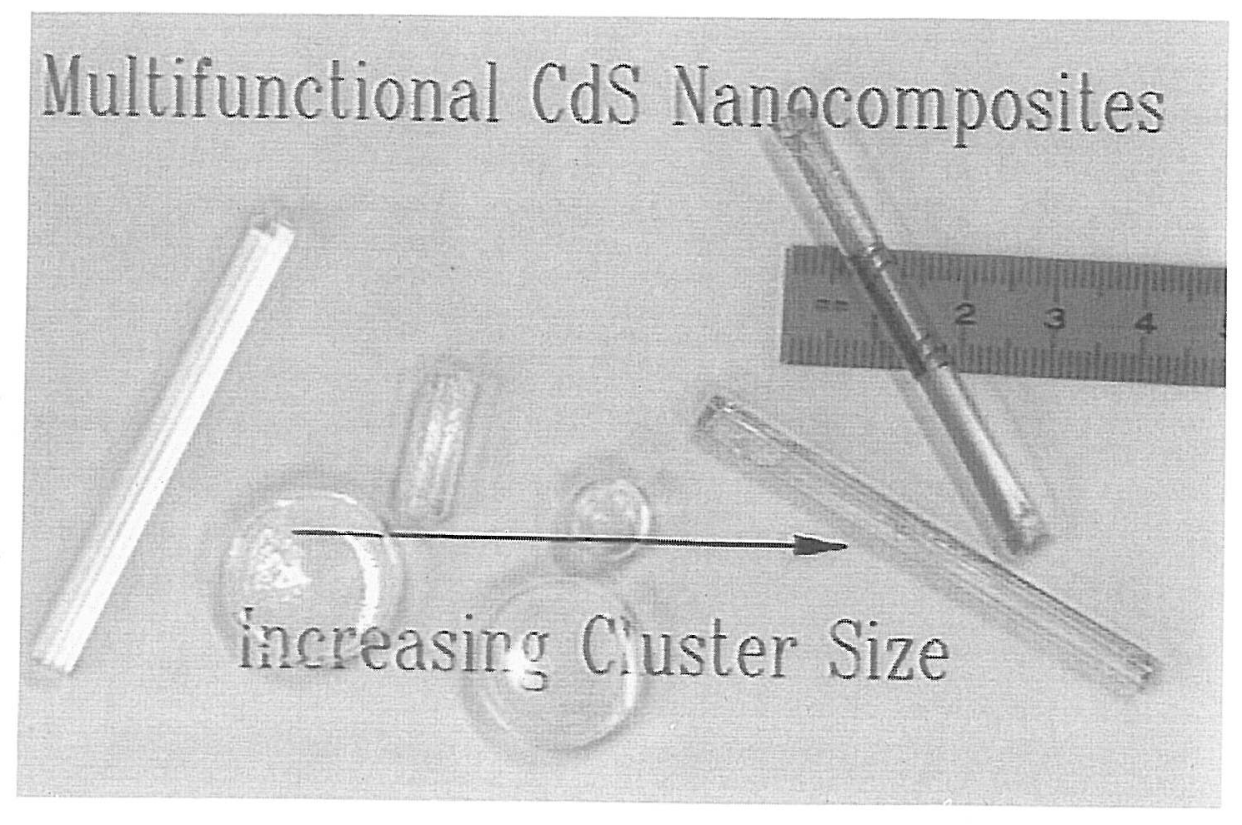

Fig. 6. Quantum dot CdS nanocomposites of different shapes and cluster sizes. 
be applied additionally. This also opens up the possibility of further optimization of the synthesis routes and of the electronic properties.

The authors want to thank Dr H. Krug for his helpful discussions and $\mathrm{Mr} \mathrm{M}$. Schuler for carrying out the laser light scattering and TEM measurements, and the State of Saarland, Germany, for the financial support.

\section{References}

[1] A.I. Ekimov and A.A. Onushchenko, Pis'ma Zh. Eksp. Teor. Fiz. 34 (1981) 363

[2] L.E. Brus, J. Chem. Phys. 79 (1983) 5566.

[3] S. Schmitt-Rink, D.A.B. Miller and D.S. Chemla, Phys. Rev. B35 (1987) 8113.

[4] R.K. Jain and R.C. Lind, J. Opt. Soc. Am. 73 (1983) 647.

[5] A. Henglein, Top. Curr. Chem. 143 (1988) 115.

[6] M. Bawendi, M. Steigerwald and L.E. Brus, Ann. Rev. Phys. Chem. 41 (1991).

[7] L. Spanhel, M. Haase, H. Weller and A. Henglein, J. Am. Chem. Soc. 109 (1987) 5649.
[8] G. McLendon, K.J. Conklin, R. Corvan, K. Johansson, E. Magner, M. O'Neil, K. Pardue, J.S. Rogalsky and D. Whitten, in: Photochemical Energy Conversion, ed. J.R. Norris and D. Meisel (Elsevier, Amsterdam, 1989) p. 47.

[9] P. Kamat, N. Dimitrijevic and R.W. Fessenden, J. Phys. Chem. 92 (1988) 2324.

[10] Y. Wang and N. Herron, J. Phys. Chem. 95 (1991) 525.

[11] L. Spanhel and M.A. Anderson, J. Am. Chem. Soc. 112 (1990) 2278; 113 (1991) 2826.

[12] M. Nogami, M. Watabe and K. Nagasaka, SPIE (Sol-Gel Optics 1328 (1990) 119.

[13] H. Kawaguchi, T. Miyakawa, N. Tan-no, Y. Kobayashi and Y. Kurokawa, Jpn. J. Appl. Phys. 30 (1991) L 280.

[14] H. Schmidt, in: Proc. Eurogel 1991, 2nd European Conf. on Sol-Gel Technology, Saarbrücken, June 1991 (North-Holland, Amsterdam) in press.

[15] R. Nass, H. Schmidt and E. Arpac, SPIE (Sol-Gel Optics) 1328 (1990) 258.

[16] R. Kasemann, S. Brück and H. Schmidt, in: Proc. Eurogel 1991, 2nd European Conf. on Sol-Gel Technology, Saarbrücken, June 1991 (North-Holland, Amsterdam) in press.

[17] E. Martin and R. Griswold, Europlan patent no. EP274100 (1988)

[18] E. Martin US patent no. 4,725,630 (1988). 